\title{
HIGH-SPEED INTERACTION ROD AND SEGMENTED PROJECTILES INTO MASSIVE OBSTACLES
}

\author{
E.N. Kramshonkov ${ }^{1, *}, A . V$. Krainov $^{1}$, and P.V. Shorohov ${ }^{1}$ \\ ${ }^{1}$ National Research Tomsk Polytechnic University, 634050 Tomsk, Russia
}

\begin{abstract}
In this paper we consider issues related to numerical modelling of processes of high-speed impact effect of a consistent series of damaging elements on the massive barrier. Research is carried out by comparing the finite speed of penetration of this series at a speed of penetration of the elongated one-piece (rod) of the missile of the same mass and diameter. Examines the range of initial impact speeds from 1 to $12 \mathrm{~km} / \mathrm{s}$ taking into account such physical phenomena as fracture, melting and evaporation of the interacting bodies. The analysis showed that almost in all range of impact speeds segmented drummer is better solid elongated.
\end{abstract}

\section{Introduction}

High speed shock has been the most effective way to defeat the objects of different nature. Effectiveness of protective structures against impact devoted a lot of research. Constant search for effective means of destruction and the protection of space vehicles (missile defence, protection from dangerous space objects, etc.). In this regard, it seems necessary to study the impact in a wide range of impact speeds based on such physical phenomena as erosion, melting and vaporization of the interacting bodies - projectiles and obstacles. A special place in a number of lesion is segmented drums, the undoubted merit of which is their greater efficiency, in some situations, compared to traditional means of impact. Similar studies to destroy dangerous space objects in the form of a sphere from fragile material presented in [1], where the means of destruction used series (tandem) damaging elements, following each other.

\section{Problem statement and initial data}

Physical formulation of the problem, the results of which are analyzed in this paper is formulated as follows (see fig. 1). A series of cylindrical projectiles with initial velocity, interact with the massive obstacle. The drummers are arranged along a single axis at a certain distance from each other. The speed of the shock is directed along the symmetry

\footnotetext{
*Corresponding author: lux veritatis@mail.ru
} 
axis and the normal to the barrier. The problem is considered in the axis symmetric formulation. In this paper the results of solving two variants of the problem high-speed interaction of an elongated cylindrical and segmented projectiles with the massive obstacle in the range of impact velocities from 1 to $12 \mathrm{~km} / \mathrm{s}$. The problem is solved under the following initial data. An elongated firing pin is a cylinder of diameter $50 \mathrm{~mm}$ and length $200 \mathrm{~mm}$; segmented - four compact segment with a length of $50 \mathrm{~mm}$, with a line break between segments is $50 \mathrm{~mm}$. The material of the drummers in both cases - hard steel. Barrier - mild steel, $250 \mathrm{~mm}$ thick.

For the mathematical description of the behavior of interacting bodies the elasto-plastic model of mechanics of a continuous medium taking into account the changes of porosity of deformed materials [2]. Modeling of different aggregate States of substances is carried out by means of interpolation wide-range equation of state describing the solid, liquid and gaseous phase [3]. When calculating the destruction is taken into account the concept of their formation as by type of job and shift, and in the implementation of their joint action [4]. After the destruction, the material is modeled by the environment in the form of sand or dust is not able to resist tensile loads, but perceiving compressive and shear loads in compression. Damage to the material as shear is typically the first stage of disruption, followed by a discontinuity at the isolation type already provisioned environment. The final stage of the calculation of damage is the realization of numerical algorithm of the separation of the body into separate pieces. The point of this algorithm is as follows. In some areas totally destroyed material, where the condition of a separated fracture, new surfaces, which divides the body into separate pieces. These fragments generally move (fly) with obviously different average speed [5-10].
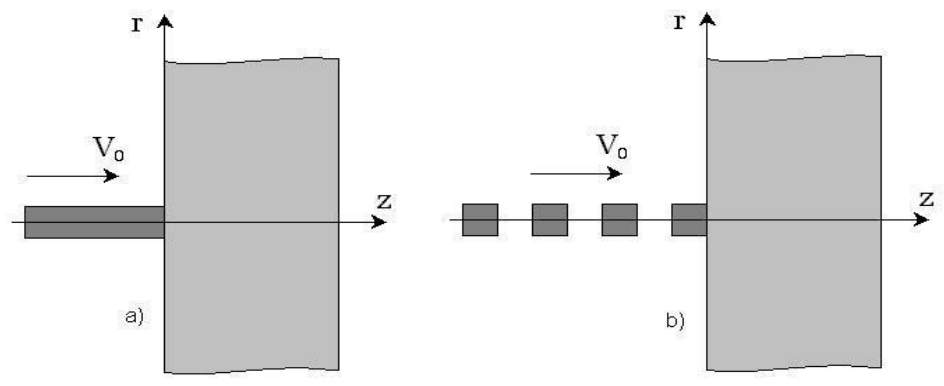

Fig. 1. The initial configuration of the penetration rod (a) and segmented (b) the drummer in the barrier.

As a research tool this study uses the method of numerical simulation based on 2-step method of second order accuracy in the Euler space variables [2, 9-13]. When calculating penetration segmented drummer applied the algorithm combining the contacting segments after actuation of interacting with the barrier segment to the size of a few computational cells [14-19]. The introduction of such algorithm is equivalent to the procedure of changing boundary conditions on the contact surface with a perfect slip condition at the rigid couplings [20-21]. Note that the use of such algorithm is possible only under the condition that all segments are made of the same material [22-25].

\section{Analysis of the results of solving the problem}

Below, as an example, it is considered two variants of the problem of the penetration rod and segmented impactor at impact velocities of $2 \mathrm{~km} / \mathrm{s}$ and $12 \mathrm{~km} / \mathrm{s}$, as well as the analysis of the efficiency of a segmented impactor compared to the rod element. At high impact 
velocities, more than $8 \mathrm{~km} / \mathrm{s}$, the efficiency of the segmented impactor decreases due to the evaporation of the front segments.In fig. 2 shows the initial stage of penetration of impactors at a speed of $2 \mathrm{~km} / \mathrm{s}$ at the time moment $t=40$ microseconds. In the upper halfplane symmetry arrows indicate field of mass velocities, where the maximum length of arrows corresponds to the maximum value of the mass speed $V$ max. Maximum speed in this case is the initial velocity of the kick. In the lower half-plane represented by intervals of relative pressure $P / P \max$, the scale intervals of the pressures shown below the figure. Numerical values of $P \max$ are given in the caption to fig. 2; here shows the numerical values of $V k$ - velocity of the contact boundary (the speed of penetration of impactors), $H k$ is the depth of the crater.

Common to both options penetration is the maximum pressure in the contact border. This pattern is characteristic for the active phase of the penetration process when the average speed of the drummer or his segment than the velocity of the contact boundary. At the initial stage of penetration, one-piece cylinder has the advantage over the segmented firing pin, as the rate of penetration $V k$, and the height $H k$ of the crater (fig. 2). However, along the length of the plastically deformed portion is not segmented drummer has more potential for penetration with higher speed $V k$. This fact is confirmed by fig. 3, which shows the distribution of the mass velocity along the symmetry axis for both options penetration.

In fig. 4 shows a configuration of interacting bodies after the complete operation of both drummers, rod and segmented. In the lower half plane of symmetry designated area of devastation projectiles and obstacles. The right side of fig. 4 shows the designations of the various types of damage to the material: SHEAR - the material is destroyed on the shift type; a SPALL - material destroyed by the isolation type; the characters "break down" correspond to different degrees of atomization of the ruined material to the relative densities of 0.5 and 0.1 . In the figure caption added numerical values of the $V t$ - speed of a back surface barrier on the symmetry axis.
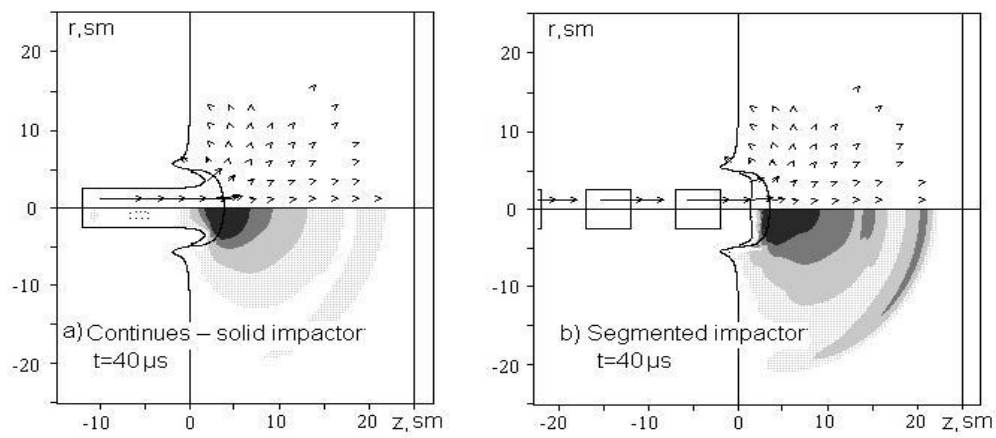

(a) $P \max =6.8 \mathrm{GPa}, V k=0.9 \mathrm{~km} / \mathrm{s}, H k=3.8 \mathrm{sm}$; (b) $P \max =2.8 \mathrm{GPa}, V k=0.7 \mathrm{~km} / \mathrm{s}, H k=3.5 \mathrm{sm}$ The scale of relative pressure intervals $P / P \max$ :
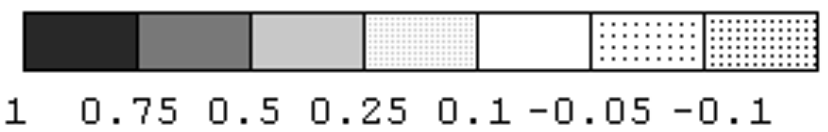

Fig. 2. The initial stage of penetration of impactor at an impact speed of $2 \mathrm{~km} / \mathrm{s}$. 

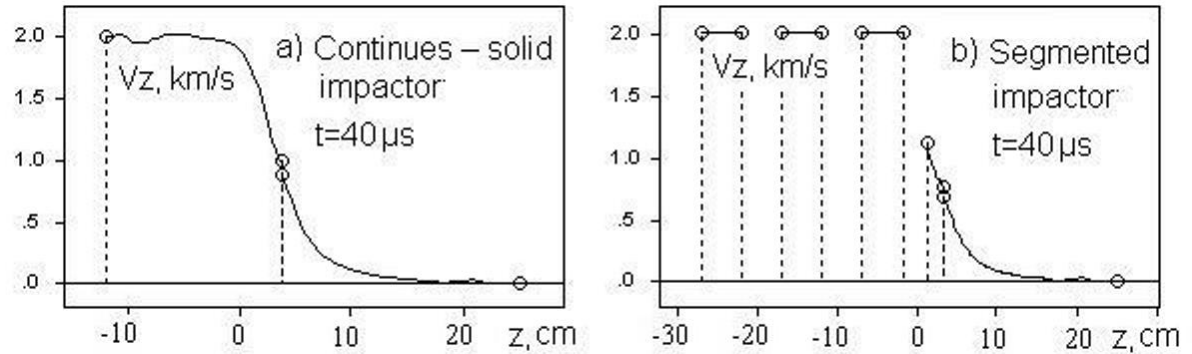

Fig. 3. The distribution of mass velocity along the symmetry axis.
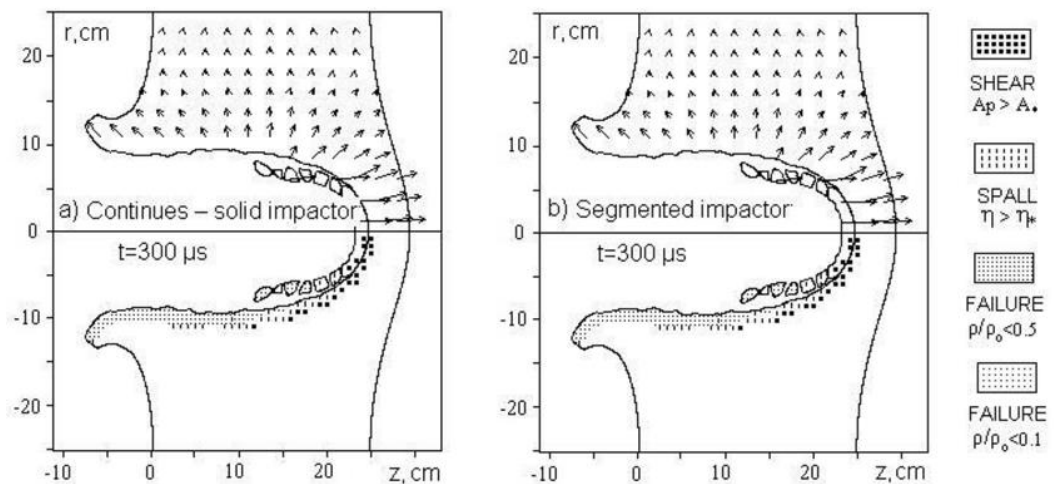

Fig. 4. The area of destruction after activation drummers: (a) $V k=0.49 \mathrm{~km} / \mathrm{s}, H k=24.7 \mathrm{~cm}, V t=0.32$ $\mathrm{km} / \mathrm{s}$; (b) $V k=0.84 \mathrm{~km} / \mathrm{s}, H k=24.1 \mathrm{~cm}, V t=0.44 \mathrm{~km} / \mathrm{s}$.

On the numerical values of the quantities $V k$ and $V t$ shows that by time $t=300 \mu \mathrm{s}$ occurs higher efficiency segmented drummer compared to the rod element. The depth of the crater $H k$ in both cases, the shock is almost aligned, in a period of $300 \mu \mathrm{s}$, this value was more core element. In fig. 5 shows the velocity dependence of the contact boundary of $V k$ and the back side of the obstacles $V t$ from the process time. For a truss element (fig. 5,a) is the characteristic stationary phase of penetration with almost constant speed $V k=900$ $\mathrm{m} / \mathrm{s}$, which continues in the period from $30 \mu \mathrm{s}$ to $150 \mu \mathrm{s}$. Saw-tooth character of the curve $V k$ in fig. $5 \mathrm{~b}$ reflects the processes of penetration of 4 elements segmented drummer with some pauses in time.
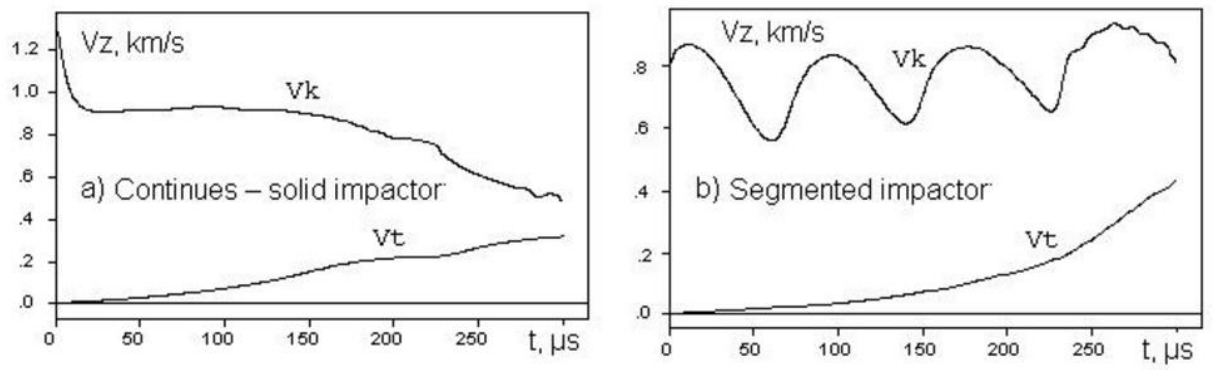

Fig. 5. The dependence of the velocity of the contact boundary of $V k$ and the back side of the obstacles $V t$ from the process time: (a) for the truss element; (b) for segmented drummer.

The final picture breaking through barriers is illustrated in figure 6 . Here it has formed a final speed breakthrough $V k$, which is the average speed of the fragments of the hammer 
and broken through the field of obstacles. Numerical values confirm his speed for greater efficiency segmented drummer compared to the rod element.
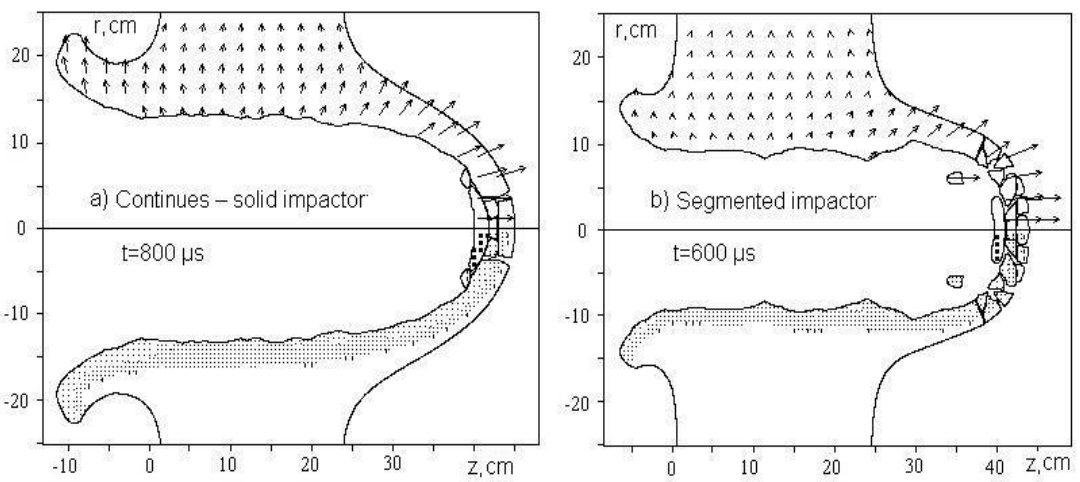

Fig. 6. The final stage of impactors penetration: a) $V k=300 \mathrm{~km} / \mathrm{s}$; b) $V k=510 \mathrm{~m} / \mathrm{s}$.

In fig. 7 shows the initial stage of penetration of impactors in the velocity of the shock interaction, is equal to $12 \mathrm{~km} / \mathrm{s}$. Here we have introduced the additional symbol regions of the state of the material, and below the figure summarizes the decoding of these States: MELTING - the internal energy of the medium is higher than energy of melting; P.GAS internal energy of the material is higher than the energy of sublimation; GAS - substance is evaporated in the state. In this embodiment, the shock also still has the advantage in the intensity of penetration of the core element, as the rate of penetration $V k$, and the depth of the crater $H k$. The entire volume of the crater the core element are filled with the vaporized material of the impactor, and the temperature on the surface of the crater reaches $150000^{\circ} \mathrm{C}$. The distribution of pressure and mass velocity along the symmetry axis are illustrated in figure 8. Given the fact that after unloading of the material is its internal energy decreases, the sublimated material may move either in the evaporated state or in the molten material. Therefore, the shock-compressed material is evaporated, its internal energy after unloading must remain higher than the energy of sublimation.
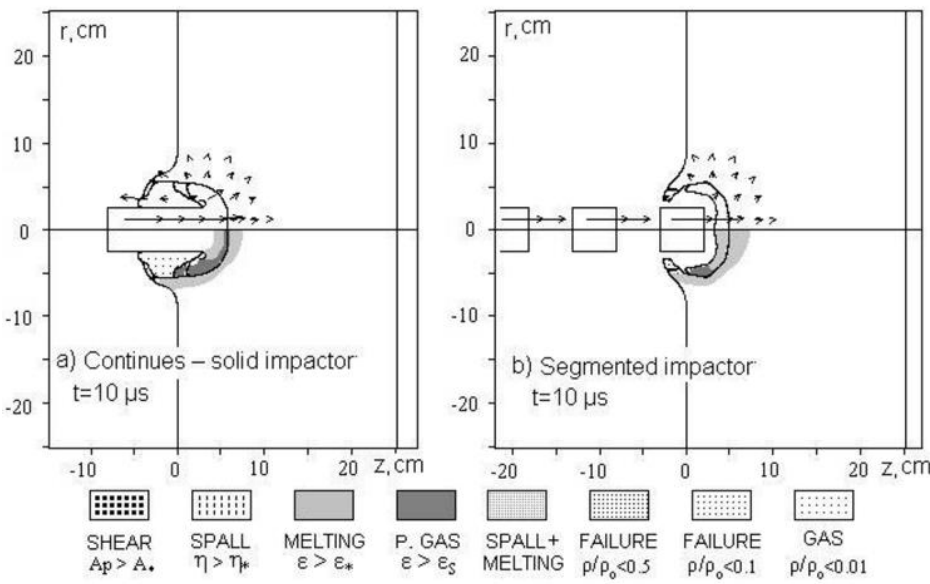

Fig. 7. The initial stage of penetration of impactor at an impact speed of $12 \mathrm{~km} / \mathrm{s}$ : (a) $P \max =201$ GPA, $V k=5.7 \mathrm{~km} / \mathrm{s}, H k=5.8 \mathrm{~cm}$; (b) $P \max =97 \mathrm{GPA}, V k=2.9 \mathrm{~km} / \mathrm{s}, H k=4.9 \mathrm{~cm}$. 

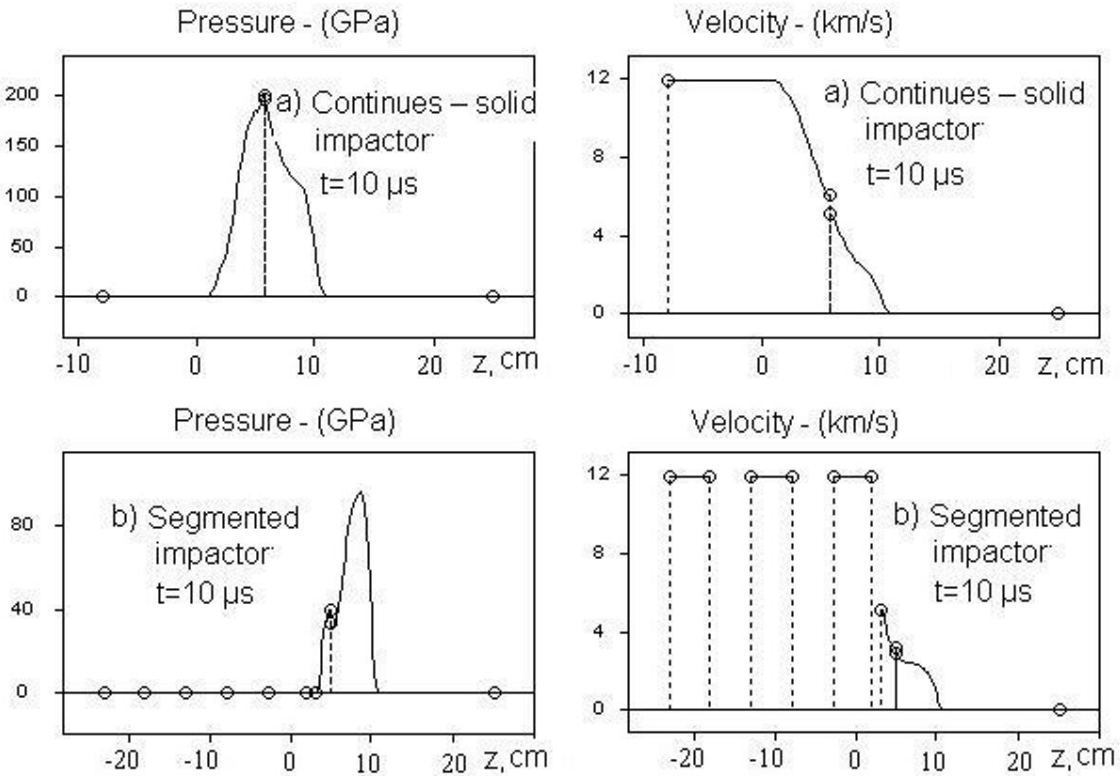

Fig. 8. The distribution of pressure and mass velocity along the symmetry axis.

Further evaporation of the materials the drums are presented in fig. 9. This stage of the process of penetration like an explosion inside the crater, vaporized substance drummers begins to scatter in the opposite direction with velocities of order $10 \mathrm{~km} / \mathrm{s}$ (fig. 9, a). The active period of the penetration of the truss element is more intense than in segmented drummer. By the time 30 ISS truss element forms a deeper crater; more intensively scatters a cloud of vaporized material of the impactor; the region of high pressures (above $100 \mathrm{HPa}$ ) is shifted to the rear side of obstacles. However, by this time the penetration speed segmented drummer Vk higher than that of the core element, and the rear segment has not yet started any direct penetration into the barrier. The rear element of the segmented impactor (fig. 9, b) penetrates through a gaseous cloud formed by evaporation from the anterior segments.
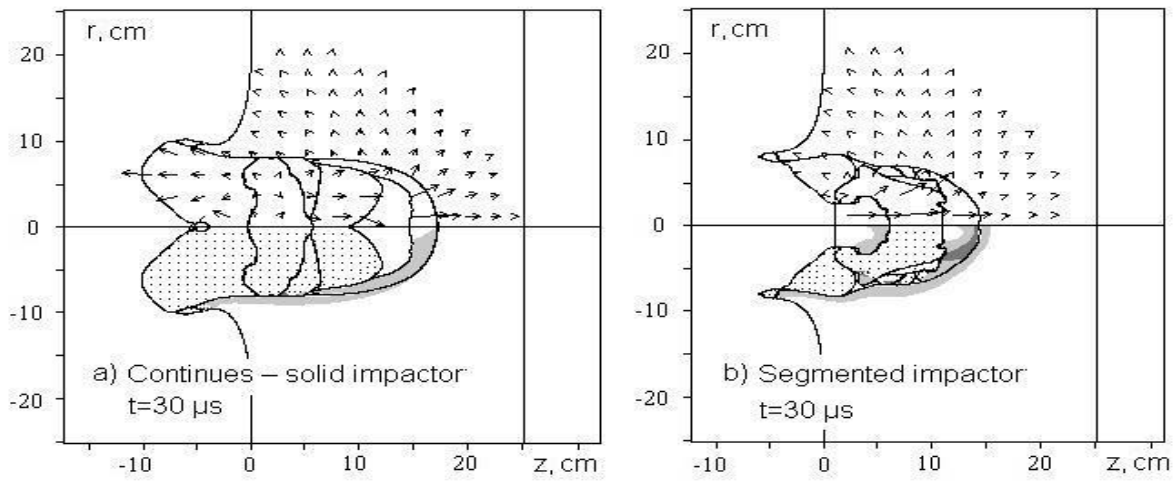

Fig. 9. Evaporation and expansion of the material of the drummer: (a) $P \max =147 \mathrm{GPa}, V \max =10.2$ $\mathrm{km} / \mathrm{s}, V k=4.5 \mathrm{~km} / \mathrm{s}, H k=17.1 \mathrm{~cm}$; (b) $P \max =202 \mathrm{GPa}, V \max =20.9 \mathrm{~km} / \mathrm{s}, V k=6.0 \mathrm{~km} / \mathrm{s}, H k=$ $14.3 \mathrm{~cm}$. 
However, he is deformed and even partially transferred in the molten state, and the cloud of gas re it accelerates to velocities of order $20 \mathrm{~km} / \mathrm{s}$ (fig. 10, b). This situation significantly reduces the efficiency of penetration of this type of firing pin under high impact velocities.
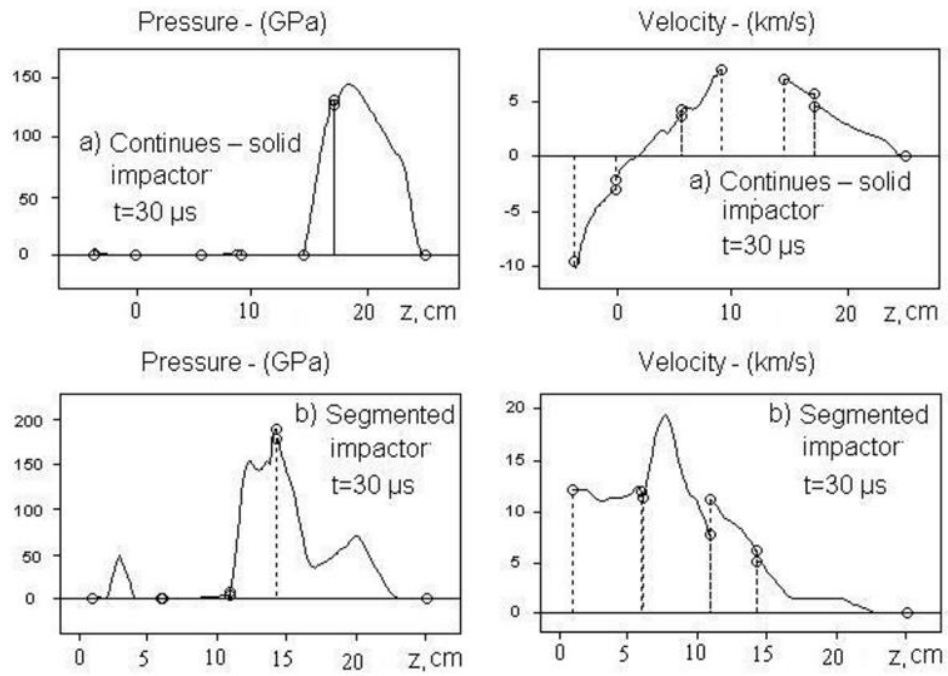

Fig. 10. The distribution of pressure and mass velocity along the symmetry axis.
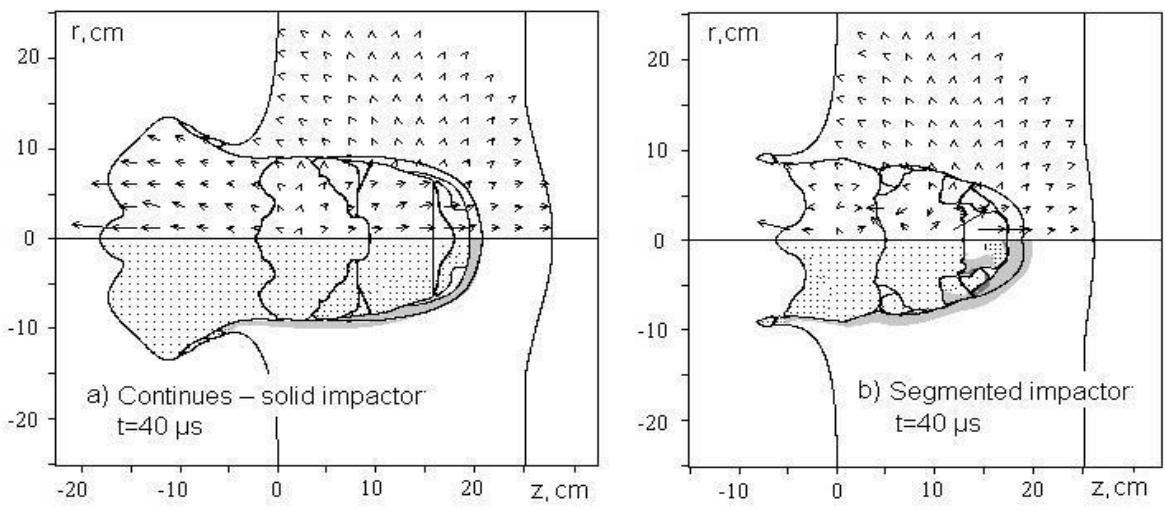

Fig. 11. The scattering of evaporated materials drummers and their penetration into the barriers: (a) $P \max =45 \mathrm{GPa}, V \max =18.0 \mathrm{~km} / \mathrm{s}, V k=2.7 \mathrm{~km} / \mathrm{s}, H k=20.7 \mathrm{~cm}$; (b) $P \max =84 \mathrm{GPA}, V \max =20.0$ $\mathrm{km} / \mathrm{s}, V k=2.9 \mathrm{~km} / \mathrm{s}, H k=18.9 \mathrm{~cm}$.

To the time moment $t=40 \mu$ s active phase of penetration of the rod element is moved to the stage of inertial motion (fig. 11, a). Speed back side barriers was higher than the speed of the contact border (fig. 12, a). This fact promotes the formation of separated region of destruction in the zone of penetration of a target (see fig. 13, a). For a segmented impactor (fig. 11, b) a process of active influence had not ended; the rear segment of drummer, located in the solid-molten state continues to penetrate the barrier, and the rate of penetration $V k$ higher than that of the core element and above the speed of the back side of the obstacles (fig. 12, b). 

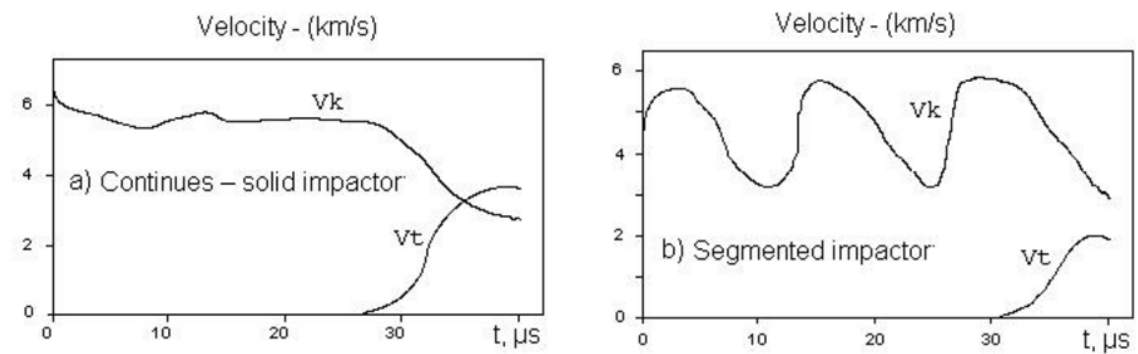

Fig. 12. The dependence of the velocity of the contact boundary of $V k$ and the back side of the obstacles $V t$ from the process time: (a) for the truss element; (b) for segmented drummer.

The final stage of penetration of impactors shown in fig. 13, illustrates the condition of the punched sections of obstacles and provides a quantitative assessment of the penetration velocity Vk. The segmented drummer this rate is almost two times higher than that of the core element. However, the total mass supergranny fragments from the core more than the segmented drummer, but they fly at a slower speed.
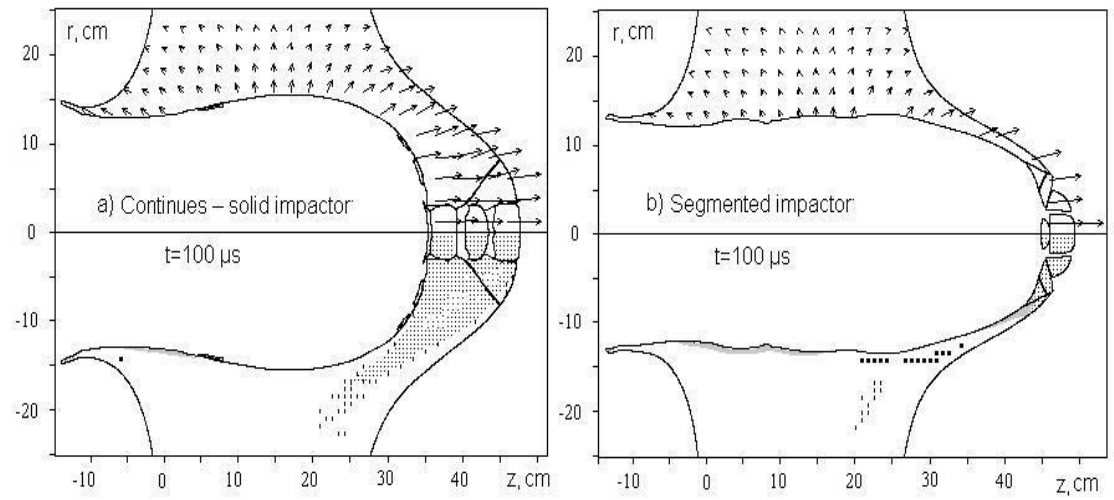

Fig. 13. The final stage of penetration of impactors: a) $V k=2.4 \mathrm{~km} / \mathrm{s}$; b) $V k=4.1 \mathrm{~km} / \mathrm{s}$.

End speed through breakthrough $V k$ is the main quantitative characteristic of the efficiency of penetration of the considered types of projectiles into a given barrier. As a result of numerical calculations in all range of impact velocities from 1 to $12 \mathrm{~km} / \mathrm{s}$ the obtained value of this quantity. From these data, calculate the coefficient of resistance $K=\left(V_{0}-V_{K}\right) / V_{0}$ of barriers for solid rod and segmented $K c, K s$. The degree of efficiency of a segmented impactor compared to the elongated cylinder is calculated by the formula Kef $=$ $(K c-K s) / K c$.

The final curves the final velocity of the penetration for solid drummer (curve 1) and segmented (curve 2) depending on the impact velocity is shown in fig. 14, there is shown a curve of the degree of efficiency (curve 3). The main conclusion from these results is that segmented the drummer is more effective compared with extended rod for the whole range of impact velocities. The efficiency of the segmented impactor increases with increasing velocity of impact in the range of impact velocities from $2 \mathrm{~km} / \mathrm{s}$ to $8 \mathrm{~km} / \mathrm{s}$. At ultra high speeds, more then $10 \mathrm{~km} / \mathrm{s}$, the value of Kef is not significantly reduced. This is due to the evaporation of the front segments and the dispersal of the vaporized substance in the opposite direction. At high impact velocities $(>6 \mathrm{~km} / \mathrm{s})$ formula for Kef only partially describes the effectiveness of both types of drummers. In this region, the hydrodynamic law of penetration, and percussionists spread over the surface of the holes, so the final speed of 
penetration, in this case, it is possible to speak as about the average speed of the front portions of the drummer and obstacles.

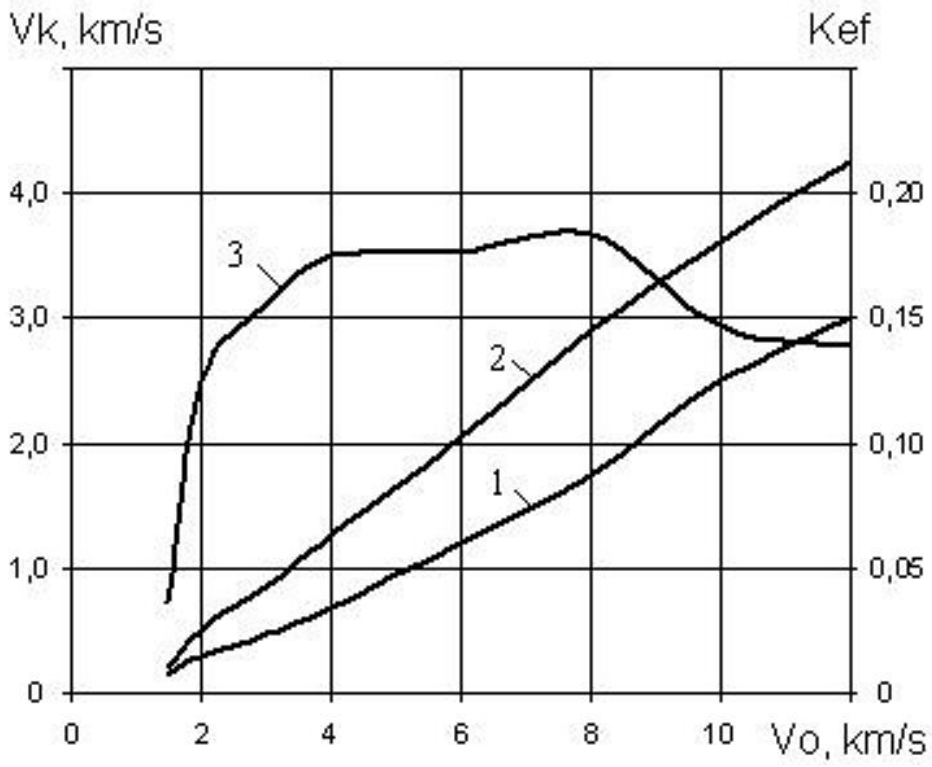

Fig. 14. Dependences of velocities breakthrough $V k$ (curves 1 and 2 for core and segmented drummer) and effectiveness $\mathrm{Kef}$ (curve 3 ) from the initial velocity of impact $\mathrm{V}_{0}$.

\section{References}

1. V.G. Trushkov, E.N. Kramshonkov, E.A. Jagnitsina, J. News of high schools. Phys. 194 (2007)

2. V.G. Trushkov, Numerical investigation of high-speed interaction objects of large deformation medium in the Euler space variables (2007)

3. A.V. Zhukov, J. Def. Solid Mech. 70 (1987)

4. V.F. Tolkachyov, V.G. Trushkov, J. Chem. Phys. 2 (1993)

5. L.V. Efremova, A.I. Korneev, V. G. Trushkov, J. Phys. Comb. Expl. 2 (1987)

6. C.V. Razorenov, G.I. Kanel, E.N. Kramshonkov, K. Baumung, J. Phys. Comb. Expl. 5 (2002)

7. A. V. Kraynov, G. V. Kuznetsov, Web Conf. 76 (2014)

8. A.V. Kraynov, E.N. Pashkov, A.V. Ponomarev, J. Adv. Mat. Res. 1040 (2014)

9. G.V. Kuznetsov, A.V. Krainov, J. Appl. Mech. Tech. Phys. 5 (2001)

10. G.V. Kuznetsov, A.V. Krainov, J. High Temp. 2 (2003)

11. A.V. Kraynov, V.A. Arhipov, EPJ Web Conf. 110 (2016)

12. A.V. Kraynov, E.N. Pashkov, R.E. Lushnikov, V.A. Arkhipov, J. Key Eng. Mat. 685 (2016)

13. E.N. Kramshonkov, A.V. Kraynov, P.V. Shorokhov, EPJ Web Conf. 110 (2016)

14. H. Park, H. Kim, Int. J. Imp. Eng. 37 (2010)

15. P.M. Booker, J.D. Cargile, B.L. Kistler, V.La Saponara, Int. J. Imp. Eng. 36 (2009)

16. H. Wu, Q. Fang, Y. Peng, Z.M. Gong, X.Z. Kong, Int. J. Imp. Eng. 76 (2015)

17. Y.Y. Zhang, L. Chen, Int. J. Imp. Eng. 36 (2009) 
18. T.T. Garfield, W.D. Richins, T.K. Larson, C.P. Pantelides, J.E. Blakeley, ICM'11 10 (2011)

19. R.T. Moura, A.H. Clausen, E. Fagerholt, M. Alves, M. Langseth, Int. J. Imp. Eng. 37 (2010)

20. Hua Jiang, Xiaowo Wang, Shuanhai He, Mat. \& Des. 39 (2012)

21. J.J. Rimoli, B. Talamini, J.J. Wetzel, K.P. Dharmasena, R. Radovitzky, H.N.G. Wadley, Int. J. Imp. Eng. 38 (2011)

22. T. Børvik, L. Olovsson, A.G. Hanssen, K.P. Dharmasena, H. Hansson, H.N.G. Wadley, J. Mech. Phys. Sol. 5 (2011)

23. C.J. Yungwirth, J. O'Connor, A. Zakraysek, V.S. Deshpande, H.N.G Wadley, J. Amer. Cer. Soc. 94 (2011)

24. Hamid Gheadnia, Ozdes Cermik, Dan B. Marghitu, Int. J. Imp. Eng. 86 (2015)

25. M. Scapin, L. Peroni, M. Peroni, Int. J. Imp. Eng. 40 (2012) 